\title{
Estatinas na Sepse: Nova Arma Terapêutica no Arsenal da Medicina Intensiva?*
}

\section{Statins on Sepsis: A New Therapeutic Gun in Intensive Care Medicine?}

\author{
Luiz Eduardo Fonteles Ritt' ${ }^{1}$, Helio Penna Guimarães², Álvaro Avezum³, Leopoldo Soares Piegas ${ }^{4}$
}

\section{RESUMO}

JUSTIFICATIVA E OBJETIVOS: Sepse é uma síndrome de base inflamatória; diversos fármacos que atuam sobre as vias de ativação da inflamação vêm sendo observados. As estatinas, além do conhecido efeito hipolipemiante detêm capacidades antiinflamatórias chamados pleiotrópicas. Essa ação pode ser benéfica no tratamento da sepse. O objetivo deste estudo foi revisar as principais publicações abordando o seu uso na sepse.

MÉTODO: Busca por artigos originais cruzando os unitermos sepse e estatina no MedLine entre 1990 e 2006.

RESULTADOS: Diversos estudos experimentais e retrospectivos investigaram o uso de estatinas em sepse, até o momento, em sua maioria mostraram melhora de mortalidade e morbidade. Não existem estudos prospectivos, aleatórios, placebo-controlados ou metanálises, o que demonstrou que a falta de evidências sólidas e comprovadas para indicação de uso desta conduta

1, Médico Residente do Instituto Dante Pazzanese de Cardiologia; 2. Médico da Divisão de Pesquisa do Instituto Dante Pazzanese de Cardiologia; Título de Especialista em Terapia Intensiva Adulto pela AMIB/AMB.

3. Diretor da Divisão de Pesquisa do Instituto Dante Pazzanese de Cardiologia;

4. Diretor Geral do Instituto Dante Pazzanese de Cardiologia; Título de Especialista em Terapia Intensiva Adulto pela AMIB/AMB.

*Recebido da Divisão de Pesquisa do Instituto Dante Pazzanese de Cardiologia, São Paulo, SP

Apresentado em 27 de agosto de 2006

Aceito para publicação em 16 de novembro de 2006

Endereço para correspondência:

Dr. Hélio Penna Guimarães

Av. Dr Dante Pazzanese, $500-1^{\circ}$ Andar

Ibirapuera

04012-909 São Paulo, SP

E-mail: heliopg@dantepazzanese.org.br

(CAssociação de Medicina Intensiva Brasileira, 2006 ainda é prevalente. Portanto ainda com seu beneficio questionável, são necessários estudos clínicos aleatórios que possam comprovar a teoria em questão.

CONCLUSÕES: Diversos estudos experimentais e retrospectivos têm investigado o uso de estatinas em pacientes sépticos, porém, apesar da literatura demonstrar ser esta teoria promissora, ainda necessita-se de estudos clínicos abordando maior número de pacientes, de forma aleatória, para estar-se apto a entender e, possivelmente, recomendar o uso desta medicação no tratamento de pacientes com sepse.

Unitermos: choque séptico, disfunção de múltiplos órgãos e sistemas, Estatinas, inflamação, sepse

\section{SUMMARY}

BACKGROUND AND OBJECTIVES: Sepsis is a syndrome with an important inflammatory component in its pathophysiology. Some drugs that act on the activation of inflammatory cascade have been tested in septic patients. Statins, beyond hypolipemic effects, have anti-inflammatory capacities, known as pleiotropic effects. That action may be of value on sepsis treatment. Review of publications who discuss the use of statins. This article has the objective to review the mainly papers about statins and sepses.

METHODS: Original published articles were searched using Medline database crossing the keys words "sepsis and statins" between 1990 and 2006

RESULTS: Experimental and retrospective studies has been investigated the use of statins in septic patients, until this moment, most of then show benefits on morbidity and mortality. However, there is not prospective, randomized, placebo controlled trials. Or metanalyses, witch denotes lack strong and robust that could be indicated this action. So, since the benefit is still unable, it is necessary randomized clinical trials witch can prove this theory.

CONCLUSIONS: Several experimental and retrospective studies have investigated the use of statins in septic 
patients, but despite literature demonstrating a promising role for its use in these patients, these studies are experimental or retrospective. Therefore, we must wait larger, prospective, randomized trials before we may be able to understand its role and possibly recommend these drugs in the treatment of septic patients

Key Words: inflammation, multiple organs and systems dysfunction, sepsis, septic shock, Statins

\section{INTRODUÇÃO}

A sepse é uma das principais causas de óbito em pacientes internados em Unidades de Terapia Intensiva (UTI) e os custos estimados para o tratamento destes pacientes são cada vez mais elevados.

A sepse pode ser definida como síndrome da resposta inflamatória sistêmica (SIRS) que ocorre em vigência de um quadro infeccioso ${ }^{1}$. Diversos mecanismos estão imbricados em sua fisiopatologia, entre eles a produção de citocinas inflamatórias, adesão e migração de monócitos e células $\mathrm{T}$, produção de óxido nítrico e apoptose.

Nos últimos anos, diversos medicamentos foram testados na prevenção e tratamento da sepse, com resultados quase sempre pouco animadores, particularmente ao se tentar debelar pontos específicos dos imbricados mecanismos de inflamação; neste desalentador cenário, apenas o uso de corticóides em baixas doses ${ }^{2}$ por suprir déficit da resposta de estresse hormonal da adrenal e Proteína $\mathrm{C}$ ativada ${ }^{3}$ demonstraram-se, até o momento, capazes de reduzir a morbimortalidade.

As estatinas são medicações tradicionalmente já consagradas na Cardiologia e Endocrinologia para o tratamento da dislipidemia; têm sua ação por meio da inibição da enzima HMG-CoA redutase, propiciando a redução da síntese de mevalonato e, por conseguinte, a produção de colesterol ${ }^{4}$.

As estatinas reduzem a mortalidade em pacientes com aterosclerose $e^{5,6}$, inclusive com evidências recentes de redução do volume da placa aterosclerótica ${ }^{7}$ e controle dos mecanismos de inflamação associados à gênese de ateroma ${ }^{8}$. Acredita-se, que não apenas a redução do colesterol sérico,mas também ações antiinflamatórias das estatinas, chamados efeitos pleiotrópicos, podem ser responsáveis por esses relevantes resultados com direta implicação sobre a sobrevida.

Neste contexto, em se considerando a sepse como entidade clinica grave com intenso processo de atividade inflamatória associada e reconhecendo a capacidade pleiotrópica das estatinas de intervenção nessas vias, o uso delas no tratamento da sepse vem sendo discutido e avaliado.

O objetivo deste artigo foi apresentar as principais informações até então publicadas sobre o uso de estatinas em sepse, discutindo novas hipóteses e possíveis indicações de seu uso na prática clínica.

\section{METODO}

Foi realizado um levantamento bibliográfico na base de dados MedLine para artigos publicados a partir de 1990, com os unitermos "sepse e estatina". Encontrou-se 49 artigos, sendo compilados para este estudo aqueles mais citados como referências em publicações.

\section{RESULTADOS}

\section{Mecanismos de Ação}

Em síndromes complexas como a sepse e o choque séptico diversas vias de ativação celular e cascatas humorais estão em atividade. Agir somente em um componente destas vias não consegue interromper o processo.

As estatinas podem afetar o processo inflamatório de diversas formas:

1. Agem na expressão molecular na membrana celular diminuindo a adesão, ativação e recrutamento celular (reduzem a produção de CD 11b agindo na adesão monocitária ao endotélio, inibindo diretamente o LFA 1 e reduzindo a ativação de linfócitos $T)^{9,10}$.

2. Reduzem indicadores de resposta aguda à inflamação (IL-6, PCR) ${ }^{11}$.

3. Aumentam a produção e a função da eNOS e reduzem a ação do óxido nítrico, um dos principais responsáveis pela vasoplegia nos estados de choque, particularmente no choque séptico ${ }^{12}$.

4. Reduzem também a apoptose, pois aumentam a produção do cNOS (óxido nítrico redutase constitutiva) que tem efeito antiapoptótico, reduzindo a lesão de órgão e disfunção de múltiplos sistemas ${ }^{13}$.

\section{Evidências Experimentais}

Merx e col. ${ }^{14}$ em um modelo de sepse por ligação e perfuração do ceco em cobaias, divididos em grupos placebo e uso prévio de estatina (sinvastatina $\pm 3 \mathrm{~h}$ antes do procedimento), comparando-os entre si e com controles operados, mas não submetidos à perfuração cecal, avaliaram a sobrevida, os parâmetros hemodinâmicos e a resposta à dobutamina. A sobrevida média no grupo placebo foi de $28 \mathrm{~h}$ e no grupo tratamento de 
108h ( $\mathrm{p}<0,005$ ); após $20 \mathrm{~h}$ do procedimento, o débito cardíaco diminuiu de 1,24 $\pm 0,09 \mathrm{~mL} / \mathrm{min} / \mathrm{g}$ para $0,87 \pm$ $0,11 \mathrm{~mL} / \mathrm{min} / \mathrm{g}$ nos não tratados e permaneceu inalterado $(1,21 \pm 0,08$ para $1,15 \pm 0,10)$ nos tratados. O uso de dobutamina levou o incremento do débito cardíaco nos grupos tratado e controle, mas se manteve inalterado nas cobaias do grupo placebo, sugerindo uma recuperação na capacidade da reação às catecolaminas com o uso da sinvastatina; em se avaliando a pressão arterial média após estímulo inotrópico, resultados semelhantes foram encontrados.

Este mesmo grupo de investigadores, em segundo estudo ${ }^{15}$, realizou um modelo de sepse semelhante ao anterior, mas fez uso não só da sinvastatina, como também de atorvastatina, fluvastatina e pravastatina; outra diferença foi o momento de administração do fármaco, modificado para 6 à $18 \mathrm{~h}$ após a indução da sepse. A sobrevida do grupo placebo foi de $23 \pm 1,2 \mathrm{~h}$ e no grupo atorvastatina elevou-se em $70 \%$ (39 $\pm 3,9 \mathrm{~h})$, $74 \%$ no grupo pravastatina $(40 \pm 4,2 \mathrm{~h})$ e $61 \%$ no grupo sinvastatina $(37 \pm 3,6 h), p<0,05$ versus placebo. Os camundongos tratados com fluvastatina, porém, só tiveram elevação de $17 \%$ na sobrevida, não atingindo estatística significativa. Não ficou claro o motivo ou mecanismo pelo qual a fluvastatina não se demonstrou efetiva neste cenário, denotando talvez aspectos intrínsecos de ação das diferentes estatinas avaliadas.

Também, em modelo experimental de sepse, induzido por ligação e perfuração cecal (LPC) em murinos, Yasuda e col. ${ }^{16}$ estudaram o efeito do uso de sinvastatina na incidência de lesão renal induzida por sepse; a sobrevivência no grupo tratado com fluídos e antibióticos foi de $100 \%$ (24h), 42\% (48h), $26 \%$ (72h), no grupo que, além deste tratamento, recebeu estatina, a sobrevivência foi de $95 \%, 84 \%, 73 \%$, respectivamente em 24,48 e 72 h $(p<0,05)$. Os níveis de uréia e creatinina séricas aumentaram significativamente em $6 \mathrm{~h}$, com piora em 24h nos animais submetidos à LPC; o tratamento com estatina preveniu estes efeitos em $24 \mathrm{~h}$. $\mathrm{Na}$ análise histológica a sinvastatina reduziu a lesão tubular tanto no córtex quanto na medula renal e diminuiu também a permeabilidade vascular renal induzida pela sepse.

Induzindo choque circulatório com endotoxina (LPS) em cobaias, Giuoti-Paiva e col. ${ }^{17}$ avaliaram a produção de óxido nítrico e a resposta cardiovascular à infusão de fenilefrina em grupos tratados ou não com sinvastatina em diferentes doses $(10,20,40,80 \mathrm{mg} / \mathrm{kg})$. Os níveis de óxido nítrico elevaram-se significativamente em $2 \mathrm{~h}$ após a injeção de LPS quando comparados com o grupo em que se administrou solução fisiológica, no grupo que recebeu o pré-tratamento com sinvastatina estes níveis foram reduzidos ( $p<0,001)$.A injeção de LPS produziu hipotensão arterial prolongada no grupo experimental; o pré-tratamento com sinvastatina não impediu este efeito hipotensor, por outro lado a resposta ao uso da fenilefrina foi restabelecida no grupo tratado com estatina o que não ocorreu no grupo tratado somente com solução fisiológica $(p<0,01)$.

\section{Evidências em Humanos}

Em uma das primeiras evidências do benefício do uso de estatinas em pacientes com sepse, Liappis e col. ${ }^{18}$ avaliaram, retrospectivamente, 388 internações por bacteremia ( $S$ aureus e Gram-) em hospital de Washington, entre 1995 e 2000. A mortalidade hospitalar no grupo que usou estatina foi de $6 \%$ e de $28 \%$ no grupo sem estatina ( $p=0,0002)$, com resultados mantidos mesmo após análise multivariada.

Também Kruger e col. ${ }^{19}$ realizaram uma análise retrospectiva de todas admissões por bacteremia em hospital de 300 leitos, totalizando 438 pacientes, entre os anos de 2000 e 2003; informações sobre uso prévio de estatina, desfecho em 28 dias, dados laboratoriais e clínicos foram coletados. Apesar de mais idosos e com maior número de comorbidades, o grupo de pacientes que utilizavam estatina previamente $(15 \%$ da amostra), teve mortalidade de $10,6 \%$ versus $23,1 \%$ no grupo sem estatina (RR 0,4; IC 0,17-0,9; $p<0,022$ ). Nesta análise, $85 \%$ dos pacientes que usavam estatina mantiveram seu uso intra-hospitalar. Neste estudo, o seu uso foi fator protetor independente, mesmo quando analisados somente os óbitos com infecção como causa direta.

Usando dados populacionais do Canadá, Hackam e col. ${ }^{20}$ compararam em estudo de coorte os pacientes em uso ou não de estatinas, de forma pareada. Todos os pacientes haviam sido internados entre 1997 e 2002 por evento cardiovascular ou para revascularização do miocárdio. Um total de 69.168 pacientes foi analisado, em relação ao óbito e/ou hospitalização por sepse. A prevalência de sepse no grupo que usava estatina foi de 71,2 eventos/10000 pacientes/ano versus 88 eventos/10000 pacientes/ano no grupo controle (RR 0,81; IC 0,72-0,91).

Thomsen e col. ${ }^{21}$ avaliaram os registros do banco de dados do condado de North Juthland na Dinamarca e selecionaram os pacientes internados por um primeiro episódio de bacteremia entre os anos de 1997 e 2003, totalizando 5353 pacientes dos quais $176(3,3 \%)$ haviam usado estatina no ano anterior. $O$ seguimento 
de 30 dias mostrou mortalidade de $20 \%$ no grupo que usou estatina e $21 \%$ no grupo de não usuários ( $p=N S)$, entre 31 e 180 dias a mortalidade foi de $8,3 \%$ e $17,5 \%$, respectivamente no grupo estatina e não usuários de estatina (RR 0,44 IC 0,24-0,80). Os autores concluíram que o uso prévio de estatina não alterou sobrevida de curto prazo, mas somente de longo prazo em pacientes admitidos por bacteremia.

Almong e col. ${ }^{22}$ acompanharam, prospectivamente, 361 pacientes admitidos em hospital por bacteremia (pneumonia, infecção do trato urinário e celulite); 22\% eram usuários de estatina. Foram avaliadas a necessidade de internação em UTI e a incidência de sepse nos dois grupos. A sepse grave ocorreu em 2,4\% dos usuários de estatina e em 19\% dos não usuários (RR 0,13; IC 0,03-0,52; RRR 16,6\%; p < 0,001). A admissão em UTI foi necessária para 3,7\% dos tratados e em 12,2\% dos não tratados (RR 0,30; IC 0,1-0,95; $p<0,025$ ).

Avaliando um estudo retrospectivo de 120 pacientes graves, com índice APACHE $>20$ e diagnóstico de disfunção de múltiplos órgãos e sistemas (DMOS), divididos em 2 grupos pareados para sexo e idade, com base no uso ou não de estatinas (40 no grupo estatina e 80 pacientes no controle), Schmidt e col..$^{23}$ encontraram mortalidade em 28 dias de $33 \%$ no grupo estatina versus $53 \%$ no grupo não usuários ( $p=0,03$ ). Quando se avaliou a mortalidade hospitalar total, detectou-se $35 \%$ no grupo estatina e $72 \%$ no controle ( $p$ $<0,0001)$.

Em estudo com adição de solução de estatina em amostras de sangue de humanos, Durant e col. ${ }^{24}$ analisaram a produção de ânion superóxido em três grupos de amostras: voluntários sadios, pacientes com sepse e pacientes internados sem sepse. A sinvastatina reduziu em $40 \%$ a produção dessa substância, diretamente ligada na fisiopatologia da sepse.

Infelizmente, ainda não se têm estudos prospectivo, aleatório, placebo-controlado para uma resposta definitiva a esta questão

\section{DISCUSSÃO}

Nos últimos anos as hipóteses de uso das estatinas em diversas doenças, que não a dislipidemia, vêm sendo consideradas.

Os efeitos das estatinas em pacientes sépticos possivelmente não têm relação com a redução dos níveis lipídicos $^{16} \mathrm{em}$ se considerando que outros fármacos redutores do colesterol parecem não ter efeito na sepse ${ }^{21}$. O possível efeito comum da maioria das estatinas na sepse (atorvastatina, sinvastatina, pravastatina), a despeito da fluvastatina, demonstrou-se eficaz tanto nos estudos experimentais, quanto nos retrospectivos.

Os estudos de segurança previamente realizados com as estatinas não incluíam pacientes sépticos, e obviamente não se pode extrapolar a incidências de efeitos colaterais do uso deste grupo de fármacos para pacientes graves com sepse. Considerando que o efeito colateral mais temido do uso de estatinas é a rabdomiólise, as estatinas são metabolizadas pelo fígado e graus diferentes de disfunção hepática estão presentes nestes pacientes que, somando-se à desidratação comumente associada ao quadro, poderiam facilitar a sua ocorrência. A maior incidência de efeitos colaterais não foi relatada nos estudos retrospectivos em humanos.

A despeito das progressivas evidências criadas em estudos observacionais ou em animais, as evidências expostas como principais ainda carecem de adequado método e robustez para solidificar o argumento cientifico que poderia justificar o acréscimo deste tratamento ao arsenal terapêutico da sepse

\section{CONCLUSÃO}

As evidências em favor do uso de estatinas em pacientes sépticos, apesar de originadas de estudos experimentais ou retrospectivos, em sua maioria, sugerem um efeito benéfico do uso em paciente com quadro séptico e hospitalizado. A capacidade em prevenir episódios de bacteremia também é sugerida.

No entanto, com base nos dados apresentados, não se pode, ainda, inferir a prescrição das estatinas para o tratamento de pacientes sépticos, considerando que diversos fármacos que se mostravam eficazes em modelos experimentais em sepse falharam sistematicamente em manter tais resultados quando aplicados em humanos.

Obviamente evidências advindas de estudos retrospectivos não são sólidas e robustas para indicar o seu uso rotineiro e estudos duplamente encoberto, aleatorizados e adequadamente controlado, testando a estatina neste cenário, devem ser incentivados para a resposta definitiva a esta questão.

\section{REFERÊNCIAS}

01. Surviving Sepsis Campaign Guidelines for management of severe sepsis and septic shock. Crit Care Med, 2004;32:1215-1218.

02. Annane D, Sebille V, Charpentier C et al - Effects of treatment with low doses of hydrocortisone and fludrocortisone on mortality in patients 
with septic shock. JAMA, 2002;288:862-871.

03. Bernard GR, Vincent JL, Laterre PF et al - Efficacy and safety of recombinant human activated protein $\mathrm{C}$ for severe sepsis. N Engl J Med, 2001;344:699-709.

04. Genert J, Libby P, Gotto AM - Heart Disease. $7^{\text {th }}$ Ed, Saunders 2004.

05. Cannon $\mathrm{CP}$, Braunwald E, McCabe $\mathrm{CH}$ et al - Intensive versus moderate lipid lowering with statins after acute coronary syndromes. N Engl J Med, 2004;350:1495-1504.

06. LaRosa JC, Grundy SM, Waters DD et al - Intensive lipid lowering with atorvastatin in patients with stable coronary disease. $\mathrm{N} \mathrm{Engl} \mathrm{J} \mathrm{Med,}$ 2005;352:1425-1435.

07. Nissen SE, Nicholls SJ, Sipahi I et al - Effect of very high-intensity statin therapy on regression of coronary atherosclerosis: the ASTEROID trial. JAMA, 2006:295:1556-1565.

08. Libby P, Ridker PM, Maseri A - Inflammation and atherosclerosis. Circulation, 2002;105:1135-1143.

09. Weber C, Erl W, Weber KS et al - HMG CoA reductase inhibitors decrease CD 11b dependent adhesion of monocytes to endothelium and reduce increased adhesiveness of monocytes isolated from patients with hypercholesterolemia. J Am Coll Cardiol, 1997;30:1212-1217.

10. Weitz-Schmidt G, Welzenbach K, Brinkmann V et al - Statins selectively inhibit leukocyte function antigen-1 by binding to a novel regulatory integrin site. Nat Med, 2001;7:687-692.

11. Jialal I, Stein D, Balis D et al - Effect of hydroxymethyl glutaryl coenzyme a reductase inhibitor therapy on high sensitive C-reactive protein levels. Circulation, 2001;103:1933-1935.

12. Laufs $\mathrm{U}$, La Fata V, Plutzky $\mathrm{J}$ et al - Up regulation of endothelial nitric oxide synthase by HMG CoA reductase inhibitor. Circulation, 1998;97:1129-1135.

13. Yamashita T, Kawashima S, Ohashi $Y$ et al - Resistance to endotoxin shock in transgenic mice overexpressing endothelial nitric oxide syntha- se. Circulation, 2000;101:931-937.

14. Merx MW, Liehn EA, Janssens $U$ et al - HMG CoA reductase inhibitor simvastatin profoundly improves survival in a murine model of sepsis. Circulation, 2004;109:2560-2565.

15. Merx MW, Liehn EA, Graf $\mathrm{J}$ et al - Statin treatment after onset of sepsis in a murine model improves survival. Circulation, 2005;112:117-124.

16. Yasuda H, Yuen PS, Hu X et al - Simvastatin improves sepsis-induced mortality and acute kidney injury via renal vascular effects. Kidney Int, 2006;69:1535-1542.

17. Giusti-Paiva A, Martinez MR, Felix JV et al - Simvastatin decreases nitric oxide overproduction and reverts the impaired vascular responsiveness induced by endotoxic shock in rats. Shock, 2004;21:271-275.

18. Liappis AP, Kan VL, Rochester CG et al - The effect of statins on mortality in patients with bacteremia. Clin Infect Dis, 2001;33:1352-1357.

19. Kruger P, Fitzsimmons $\mathrm{K}$, Cook $\mathrm{D}$ et al- Statin therapy is associated with fewer deaths in patients with bacteraemia. Intensive Care Med, 2006;32:75-79.

20. Hackam DG, Mamdani M, Li P et al - Statins and sepsis in patients with cardiovascular disease: a population-based cohort analysis. Lancet, 2006;367:413-418.

21. Thomsen RW, Hundborg HH, Johnsen SP et al - Statin use and mortality within 180 days after bacteremia: a population-based cohort study. Crit Care Med, 2006;34:1080-1086.

22. Almog $Y$, Shefer A, Novack V et al - Prior statin therapy is associated with a decreased rate of severe sepsis. Circulation, 2004;110:880-885.

23. Schmidt H, Hennen R, Keller A et al - Association of statin therapy and increased survival in patients with multiple organ dysfunction syndrome. Intensive Care Med, 2006;38:1248-1251.

24. Durant R, Klouche K, Delbosc S et al - Superoxide anion overproduction in sepsis: effects of vitamin E and simvastatin. Shock, 2004;22:34-39. 